EGU21-10660

https://doi.org/10.5194/egusphere-egu21-10660

EGU General Assembly 2021

(c) Author(s) 2021. This work is distributed under

the Creative Commons Attribution 4.0 License.

\title{
Detecting and assessing trends of CFCs and substitutes from IASI measurements
}

\author{
Hélène De Longueville ${ }^{1}$, Lieven Clarisse ${ }^{1}$, Bruno Franco ${ }^{1}$, Simon Whitburn ${ }^{1}$, Cathy Clerbaux ${ }^{1,2}$, \\ Claude Camy-Peyret ${ }^{3}$, and Pierre-François Coheur ${ }^{1}$ \\ ${ }^{1}$ SQUARES, Université libre de Bruxelles, Brussel, Belgium \\ ${ }^{2}$ LATMOS/IPSL, UVSQ, CNRS, Sorbonne Université, Paris, France \\ ${ }^{3}$ Institut Pierre-Simon Laplace (IPSL), UPMC, Sorbonne Université, Paris, France
}

The first Infrared Atmospheric Sounding Instrument (IASI) on the Metop satellites suite has achieved more than 13 years of continuous operation. The instrument stability and the consistency between the different instruments on the successive Metop ( $A, B$ and $C$ ) is remarkable and offer the potential to investigate trends in the concentration of various species better than with any other previous or current hyperspectral IR sounder. The low noise of IASI radiances is also such that even weakly absorbing species can be identified, on single or at least on averaged spectra. In this work we exploit the first decadal record of IASI measurements to (1) detect and monitor halogenated compounds regulated by the Montreal protocol (CFCs) or used as substitutes (HCFCs, HFCs), as well as fluorinated compounds $\left(\mathrm{CF}_{4}, \mathrm{SF}_{6}\right)$ and potentially short lived chlorine species, for which substantial emissions are suspected (2) give a first assessment of the trend evolution of these species over the 2008-2017 period covered by IASI on Metop-A. This is done by targeting various geographical areas on the globe and examining the remote oceanic and continental source regions separately. The trend evolution in the different chemical species, either negative or positive, is validated against what is observed from ground-based measurement networks. We will conclude by assessing the usefulness of IASI and follow-on mission to contribute to global measurements of ozone depleting substances. 